\title{
Structure Related Photocatalytic Properties of $\mathrm{TiO}_{2}$
}

\author{
Ju-Young Park, Changhoon Lee, Kwang-Woo Jung, and Dongwoon Jung* \\ Department of Chemistry and Institute of Basic Natural Sciences, Honkwang Lniversity, Iksan, Jeonbuk 570-749, Korea \\ "E-mail: dimgàwonkwang.ac.kr \\ -Department of Chemistry, Nom Carolina State University, Raleigh, NC. 27695-8204, LSA \\ Received December 8, 2008, Accepted December 29, 2008
}

\begin{abstract}
The band structures and the densities of states at the Femi energy for rutile, anatase and brookite phases are investigated along with the structure-photocatlaytic relationship by using DFT method. Bands are less dispersive in anatase phase than in rutile phase, and they are almost flat in brookite phase. As a result the DOS value near the Fermi energy for brookite is highest among three types of $\mathrm{TiO}_{3}$, which means that the numbers of electrons near the Fermi energy are largest in brookite. The calculation shows that brookite phase may exhibit highest photocatalytic efficiency among three types of $\mathrm{TiO}_{2}$
\end{abstract}

Key Words: $\mathrm{TiO}_{2}$, Brookite. DOS. Photocatalyst

\section{Introduction}

Titanium dioxide has three different structure types : rutile. anatase. and brookite. All of these crystalline forms of $\mathrm{TiO}_{2}$ occur in nature as mineral. but only nitile and anatase have been able to be synthesized in pure form at low temperature until recent day's. Therefore anatase and rutile phases which belong to the tetragonal crystal system are widely studied on their syntheses, characterizations and photocatalytic properties. ${ }^{\hat{-}+}$ So far anatase phase is known to exhibit higher photocatalytic efficiency compared with rutile phase. Only little efforts have been made on the synthesis. characterization and properties of brookite phase which is belonging to the orthorhombic crystal system. ${ }^{5-7}$ The poor result on brookite phase is due to the difficulty in preparing the pure brookitetype $\mathrm{TiO}_{2}$ without mixture of rutile or anatase phase. Recently, the method to synthesize pure brookite phase $\mathrm{TiO}_{2}$ was reported. ${ }^{8}$ which means that versatile studies on the brookite phase can be ignited. In this paper. the band stnictures and densities of states (DOS) near the Fermi energy for rutile. anatase and brookite phase are calculated and investigated along with the structure-photocatlaytic relationship.

\section{Calculation}

The first principle calculations were performed for the three types of $\mathrm{TiO}_{2}$ crystals within the density functional theory ${ }^{9}$ (DFT) formalism, using the Cambridge Serial Total Energy Package (CASTEP) software. ${ }^{\text {li }}$ The CASTEP software solves the Koln-Sham equations with periodic boundary conditions and the pseudopotential method as an approximation of the atomic core-valence electron interaction. while the electronic wavefunctions are expanded in a plane wave basis. The Kohn-Sham band structure underestimates systematically the band gap often by more than $50 \%$. However, the result shows a good representation especially in band dispersion. We adopted the normalized conserving pseudopotentials provided within the package and the generalized gradient density approximation of Perdew. Burke and Ernzerhof (GGA-PBE) ${ }^{j 1}$ for the exchange-correlation functional. The Brillouin zone was sampled with the Monkhorst-Pack scheme ${ }^{12}$ using a k point separation of $0.04 \AA^{-1}$ in a $6 \times 6 \times 6$ grid in reciprocal space. A plane wave energy cutoff of $500 \mathrm{eV}$ and an energy convergence limit of $1 \times 10^{-} \mathrm{eV} / \mathrm{atom}$ were used in total energy calculations. guaranteeing a high level of convergence. The adopted stnucture of nutile, anatase and brookite was tetragonal. tetragonal and orthorhombic. respectively.

\section{Results and Discussion}

The bond distances of $\mathrm{Ti}-\mathrm{O}$ and $\mathrm{O}-\mathrm{O}$ in rutile. anatase and brookite are shown in Table 1. Generally. the degree of orbital interactions within a bond decreases with increasing the bond distance. Ti-O and $\mathrm{O}-\mathrm{O}$ bond distances are longest in brookite. while those are shortest in rutile plase. It is expected, therefore, the orbital interaction is expected to be strong in nutile. but weak in brookite, which in turn results in the increase of the band width as rutile $<$ anatase $<$ brookite. Figures $\mathrm{la}$ and $\mathrm{lb}$ show the band dispersion curve and density of states (DOS) of rutile structure, respectively. Bands around the Ferni energy are strongly dispersive and the DOS value within the window of $\pm 1.0 \mathrm{eV}$ of the Fermi energy is less than $5 \mathrm{e}^{\circ} / \mathrm{eV}$, as expected with the bond distances

The calculated band gap of $2.1 \mathrm{eV}$ is somewhat smaller than the experimental band gap of $3.2 \mathrm{eV}$. which is due to the origin of the Kohn-Sham method. as mentioned in introduction. Figures $2 \mathrm{a}$ and $2 \mathrm{~b}$ demonstrate the band dispersion curve and density of states (DOS) of anatase structure. respectively. The band gap of $2.1 \mathrm{eV}$ is almost similar to that of rutile, but bands are definitely less dispersive for the anatase phase compared

Table 1. The bond distances of Ti-O and O-O in Rutile, Anatase, and Brookite

\begin{tabular}{ccc}
\hline & Ti-O $(\AA)$ & $\mathrm{O}-\mathrm{O}(\AA)$ \\
\hline Rutile & $1.91-1.94$ & 2.43 \\
Anatase & $1.92-1.95$ & 2.43 \\
Brookite & $1.87-2.04$ & 2.49 \\
\hline
\end{tabular}


(a)

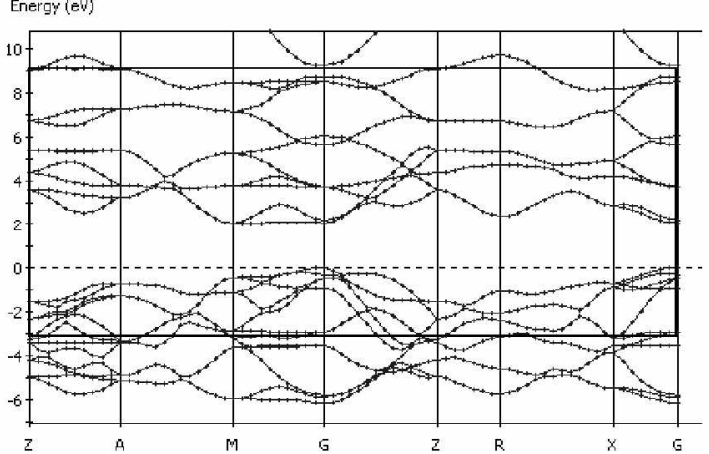

(b)

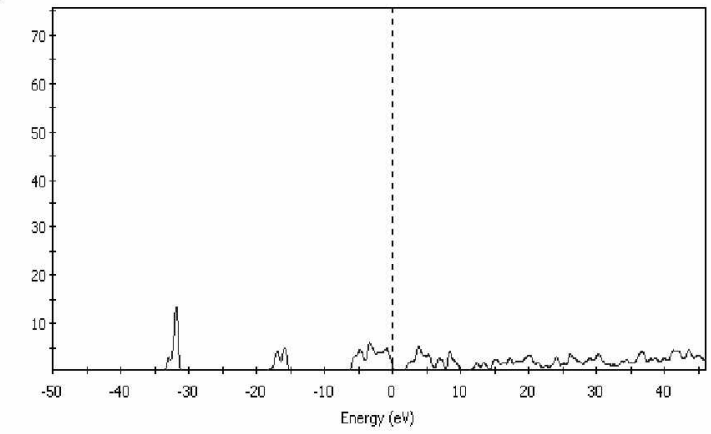

Figure 1. Calculated (a) band dispersion curve and (b) Density of States of nutile TiO, G X M Z R, A represent $(0.0,0.0,0.0)$, $(0.0,0.0,0.5),(0.5,0.5,0.0),(0.0,0.0,0.5),(0.5,0.0,0.5),(0.5$. $0.5,0.5$ josition, respectively, in the reciprocal lattice. The horizontal dashed line in dispersion curve and the vertical dashed line in DOS represent the Fermi energy.

(4)

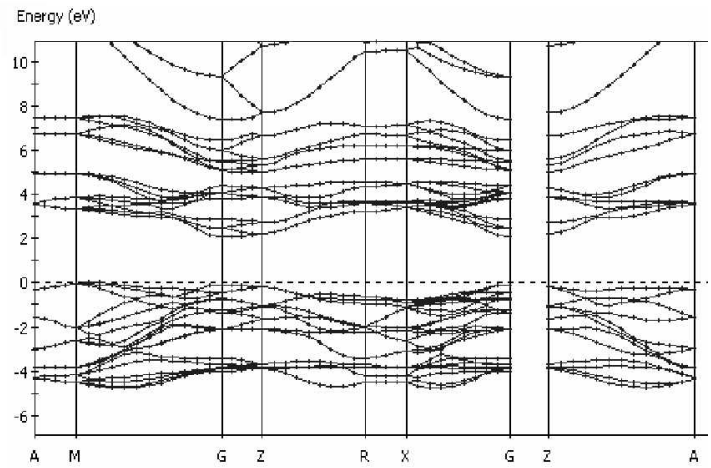

(b)

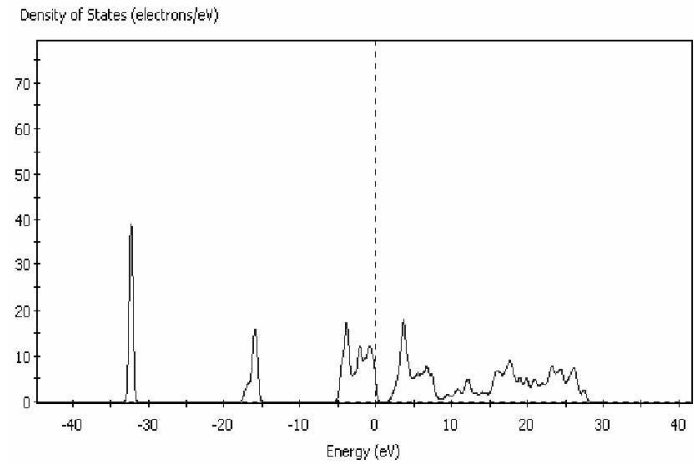

Figure 2. Calculated (a) band dispersion curve and (b) Density of States of antase $\mathrm{TiO}_{2}, \mathrm{G}, \mathrm{X}, \mathrm{M}, \mathrm{Z}, \mathrm{R}, \mathrm{A}$ represent $(0.0,0.0,0.0)$, $(0.0,0.0,0.5),(0.5,0.5,0.0),(0.0,0.0,0.5),(0.5,0.0,0.5), 10.5$, $0.5,0.5$ ) position, respectively, in the reciprocal lattice. The horzontal dashed line in dispersion curve and the vertical dashed line in DOS represent the Fermi energ! with rutile phase. thereby exhibiting ligher DOS value of about $15 \mathrm{e} / \mathrm{eV}$ within the window of $\pm 1.0 \mathrm{eV}$ of the Fermi energy. This means that anatase phase possesses larger number of electrons around the Fermi energy. The photocatalytic activity arises from the hydroxyl radicals and peroxide ions prepared through the following reactions:

1. $\mathrm{TiO}_{2}+\mathrm{h} \rightarrow \mathrm{TiO}_{2}\left(\mathrm{e}^{\cdot \mathrm{CB}}+\mathrm{h}^{+} \mathrm{VB}\right)$

2. $\mathrm{TiO}_{2}\left(\mathrm{~h}^{+} \mathrm{VB}+\mathrm{e}^{\cdot \mathrm{CB}}\right) \rightarrow \mathrm{TiO}_{2}$ (recombination)

3. $\mathrm{TiO}_{2}\left(\mathrm{~h}^{+} \mathrm{VB}\right)+\mathrm{OH}^{-} \rightarrow \mathrm{TiO}_{2}+\mathrm{OH}$

4. $\mathrm{TiO}_{2}\left(\mathrm{e}^{-\mathrm{CB}}\right)+\mathrm{O}_{2} \rightarrow \mathrm{TiO}_{2}+\mathrm{O}_{2}$.

$\mathrm{CB}$ : conduction band of $\mathrm{TiO}_{2}$

$\mathrm{VB}$ : valence band of $\mathrm{TiO}_{2}$

The degree of photocatalytic activity of $\mathrm{TiO}_{2}$ is proportional to the number of electrons enitted by ultraviolet energy. The probability of electron emission is strongly related to the electron density near the Fermi energy. Therefore the higher photocatalytic efficiency of anatase phase compared with rutile phase is understood.

Figures $3 \mathrm{a}$ and $3 \mathrm{~b}$ exhibit the band dispersion curve and density of states (DOS) of brookite structure. respectively: The band gap of $1.7 \mathrm{eV}$ is somewhat less than those of anatase and rutile. Surprisingly, bands are almost flat and the DOS value within the window of $\pm 1.0 \mathrm{eV}$ of the Fermi energy in brookite-type $\mathrm{TiO}_{2}$ is bigger than that in nutile or even in anatase phase. The photocatalytic efficiency of the material depends on the crystallinity of the material. surface area. shape of the particle. band gap. and the amount of electrons around

(a) Energy (e)

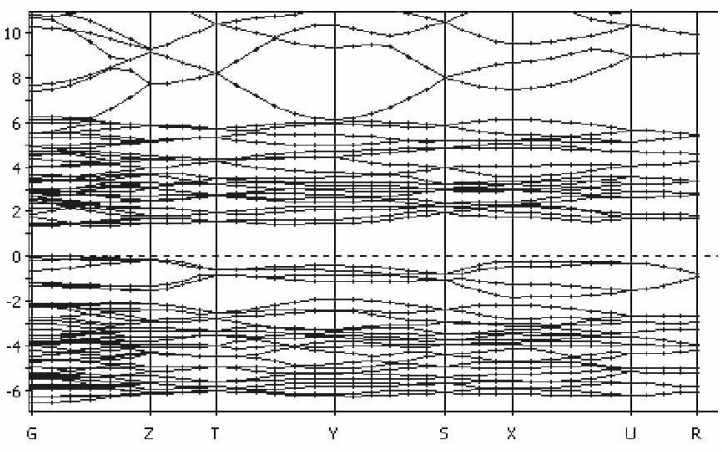

(b) Density of States (electronsiev)

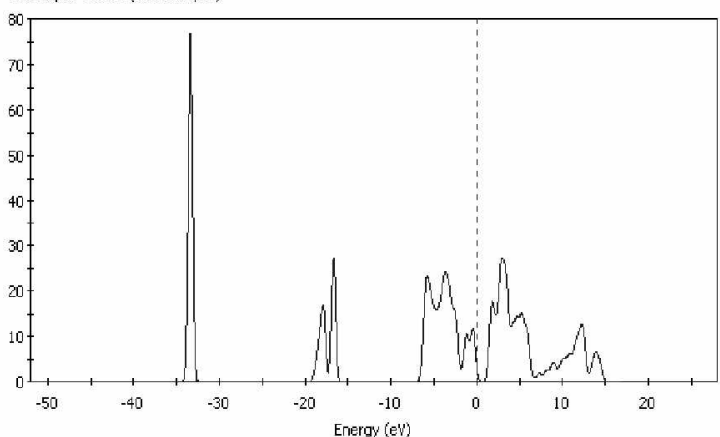

Figure 3. Calculated (a) band dispersion curve and (b) Density of States of brookite $\mathrm{TiO}_{3}$. G, X, T, Y, S, U, Z represent $(0.0,0.0$, $0.0),(0.0,0.0,0.5),(-0.5,0.0,05),(-0.5,0.0,0.0),(-0.5,0.5,0.0)$, $(0.0,0.5,0.5),(0.0,00,0.5)$ position, respectively, in the reciprocal lattice. The horizontal dashed lue in dispersion curve and the vertical dashed line in DOS represent the Femni energy. 
the Fermi energy. The calculated DOS results suggest that the photocatalytic efficiency of highy crystallized brookite phase $\mathrm{TiO}_{2}$ might be better than that of anatase when the structural conditions are similar. Not only experiments on the syntheses and characterizations of doped $\mathrm{TiO}_{2}^{13-14}$ but also developments of new materials such as brookite phase should be followed.

\section{Conclusion}

Band structures and DOS near the Fermi energy for rutile. anatase and brookite phase are calculated and investigated. The band width near the Fermi energy is smallest in the brookite-type $\mathrm{TiO}_{2}$. and therefore the DOS value near the Fermi energy is largest in the brookite-type $\mathrm{TiO}_{2}$. This means that the electronhole separation can arise most actively in brookite phase. Consequently, it is expected the brookite-type $\mathrm{TiO}_{2}$ is expected to exhibit the highest photocatalytic efficiency among three types of $\mathrm{TiO}_{2}$ when the structural conditions are similar.

Acknowledgments. This work was supported by Wonkwang University through the program of Wonkwang Research Grant of 2007.

\section{References}

1. Zheng, Y; Shi, E.; Chen, Z; Li, W.; Hu, X. J. Mater: Chem. $2001,11,1547$.

2. Kim S. T.; Park, S. D.; Jeong, Y. H. J.Am Ceram. Soc. 1999, 82, 927.

3. Yang, Y.: Mei, S.: Ferreira, I. M. J. Am. Ceram Soc 2000, 83 . 1361 .

4. Zheng, Y.: Shi, E:; Chi, S.: Li, W.: Hu, X. J. Am Ceram Soc. $2000,83,2634$.

5. Pottier, A.; Chaneac, C.; Tronc, E.; Mazerolles, L.; Jolivet, I. J. Mater. Chem 2001, 11, 1116

6. Zheng, Y.; Shi, E.: Chi, S.; Li, W.: Hu, X. J. Mater Sci. Lett. 2000,1445 .

7. Kominami, H.: Kohno, M.; Kera, Y. J. Mater Chem, 2000, 10 , 1151.

8. Lee, J. H.; Yang. Y. S. Mater: Chem. Phys. 2005, 93, 237.

9. Kohn, W.; Sham, L. T. Phys Rev A 1965, 140, 1133.

10. Segall, M:; Lindan, P.: Probert, M.: Pickard, C.: Hasnip, P: Clark, S.; Pavne, M. J. Phys.: Condens. Hatter 2002, 1H, 2717.

11. Perdew, J. P; Burke, K; Enzerhof, M. Phss. Rev Lett. 1996, 77. 3865.

12. Monkhorst, J. H.; Pack, J. Phys. Rev: $B$ 1976, 13, 5188.

13. Kim, S. W.: Khan, R.: Kim, T. J.; Kim, W. H. Bull Korean Chem. Soc. 2008, 29(6), 1217.

14. Chakraborty, A. K.; Chai, S. Y; Lee, W. I. Bull. Koreon Chem. Soc. 2008, 29(2), 494 